\title{
Multifocal Tuberculosis in Immunocompetent Patients
}

\author{
Pr H. Benjelloun, Dr. K. Chaanoun", Pr. N. Zaghba, Pr. N. Yassine
}

Chu Ibn Rochd de Casablanca Rue des Hôpitaux Casablanca, Maroc Rue des Hôpitaux Casablanca, Marocoo

DOI: $10.36347 /$ sasjm.2020.v06i03.006

| Received: 12.03.2020 | Accepted: 20.03.2020 | Published: 21.03.2020

*Corresponding author: Dr. K. Chaanoun

\section{Abstract}

Original Research Article

Multifocal tuberculosis is defined as the involvement of two non-contiguous extra-pulmonary sites with or without pulmonary involvement. It is a rare but serious form that occurs mainly in immunocompromised patients. The aim of our work was to analyze the epidemiological, clinical and evolutionary features of multifocal tuberculosis in immunocompetent patients. For this we conducted a retrospective study of 67 cases of multifocal tuberculosis in immunocompetent patients, collected at the service of respiratory diseases of Ibn Rochd University Hospital of Casablanca over a period of 17 years. There are 45 women and 22 men. The average age was 32 years old. The average consultation time was 72 days. Recent tuberculosis was found in $33 \%$ of cases. The field assessment performed on all patients for possible immunosuppression was negative. Our patients had at least three associated disorders. Pulmonary tuberculosis was found in all cases. It was associated mainly with pleural involvement in $38.8 \%$ of cases, mediastinal lymph node in $23.8 \%$ of cases, peritoneal in $19.4 \%$ of cases and peripheral lymph node in $16.4 \%$ of cases. Patients were treated according to the recommendations of the national program against tuberculosis in Morocco. The evolution was favorable in $68.6 \%$ of the cases. We insist on the possibility of the occurrence of multifocal tuberculosis even in immunocompetent subjects. TB treatment should be started as soon as possible to avoid sometimes disastrous complications.

Keywords: Tuberculosis multifocal; immunocompetent; adult; extra pulmonary tuberculosis.

Copyright @ 2020: This is an open-access article distributed under the terms of the Creative Commons Attribution license which permits unrestricted use, distribution, and reproduction in any medium for non-commercial use (NonCommercial, or CC-BY-NC) provided the original author and source are credited.

\section{INTRODUCTION}

Tuberculosis remains a public health problem, especially in developing countries such as Morocco. According to estimates by the World Health Organization, the number of all forms of tuberculosis cases in Morocco was about 31,542 in 2016 [1]. Multifocal lesions of tuberculosis are rare and represent $10 \%$ of cases [2,3] and occur most often in immunocompromised patients, especially those with HIV. However, this form of tuberculosis may be of interest even to immunocompetent individuals as is the case in our series. The objective of our study was to identify the epidemiological, clinical and evolutionary peculiarities of this form of tuberculosis.

\section{PATIENTS AND METHODS}

We report a descriptive retrospective study of 67 cases of immunocompetent patients followed in the pneumology department for multifocal tuberculosis over a period of 17 years from January 2002 to September 2019. The patients had no field of immunosuppression. They all benefited from an assessment comprising an $\mathrm{HIV}$, hepatitis $\mathrm{B}$ and $\mathrm{C}$ serology as well as a renal, hepatic assessment and a primary immunodeficiency assessment. All of our patients also had good nutritional status.

The diagnosis of tuberculosis has been made in the presence of bacteriological and / or histological confirmation or a set of epidemiological, clinical, radiological and evolutionary arguments.

For each patient, we filled in a form that allowed us to collect epidemiological, clinical, radiological, bacteriological and pathological data as well as the treatments received and the evolution. Data was processed and analyzed using SPSS software.

\section{RESULTS}

In our series of 67 cases of multifocal tuberculosis, there was a female predominance of $67 \%$. The average age of the patients was 32 years old with extremes ranging from 17 to 82 years old. All our patients were vaccinated with BCG. A recent tuberculous contagion was found in $22(33 \%)$ patients. No history of tuberculosis has been found. Seventy-five percent of the patients were of low socio-economic 
status and nine patients were smokers. The average time between onset of symptoms and first visit was 72 days with extremes ranging from 2 weeks to 4 months. Fever and alteration of the general condition were found in all cases. Tuberculin intradermal reaction (TDI) in all patients was positive in $48(71 \%)$ patients.

All patients had at least three associated disorders. Pulmonary tuberculosis was found in all cases. The pulmonary lesions were of miliary type in $56.7 \%$ of cases, pneumonia in $17.9 \%$, atelectasis in $14.9 \%$ and cavern in $10.4 \%$ of cases. It was associated with pleural involvement in $38.8 \%$ of cases, mediastinal lymph node in $23.8 \%$ of cases, peritoneal in $19.4 \%$ of cases, peripheral lymph node in $16.4 \%$ of cases. Splenic tuberculosis involved ten patients. Eight patients had tuberculous cold abscess from the wall, a surgical flattening of the abscess with bank biopsy performed in 7 patients. Seven patients had uro-genital tuberculosis. Cerebro-meningeal tuberculosis (Figure 1) was observed in six patients, five of whom had tuberculous miliary disease. Six patients had tuberculous pericarditis (Figure 2) confirmed by pericardial biopsy in 2 cases. The osteoarticular tuberculosis concerned 5 patients, it is a spondylodiscite in four cases. The cavum involvement involved two patients in our series. Psoas abscess associated with spinal involvement was noted in a single patient with percutaneous drainage (Figure 3).

Patients were treated according to the recommendations of the national tuberculosis control program in Morocco which is based on a poly-antituberculosis chemotherapy combining streptomycin, rifampicin, isoniazid, pyrazinamide and ethambutol according to the $2 \mathrm{SRHZ} / 7 \mathrm{RH}$ regimen. for cases diagnosed before 2011 and 2RHZE / 7RH for those diagnosed after. This follows the changes made on this program on this date. Oral corticosteroid therapy at a dose of $1 \mathrm{mg} / \mathrm{kg} /$ day for 4 to 6 weeks has been prescribed in patients with neuro-meningeal, pericardial or miliary hypoxemic disease with gradual dose depression. The evolution was clinically, bacteriologically and radiologically favorable in $68.6 \%$ of cases. One patient had a relapsing tuberculosis two years later, undergoing relapse treatment (2SRHEZ / 1RHEZ / 5RHE). We deplored two deaths, one in a respiratory distress chart secondary to pneumothorax complicating tuberculosis miliary, the other following multi-visceral failure secondary to disseminated intravascular coagulation (DIC) and eighteen patients were lost. of view.

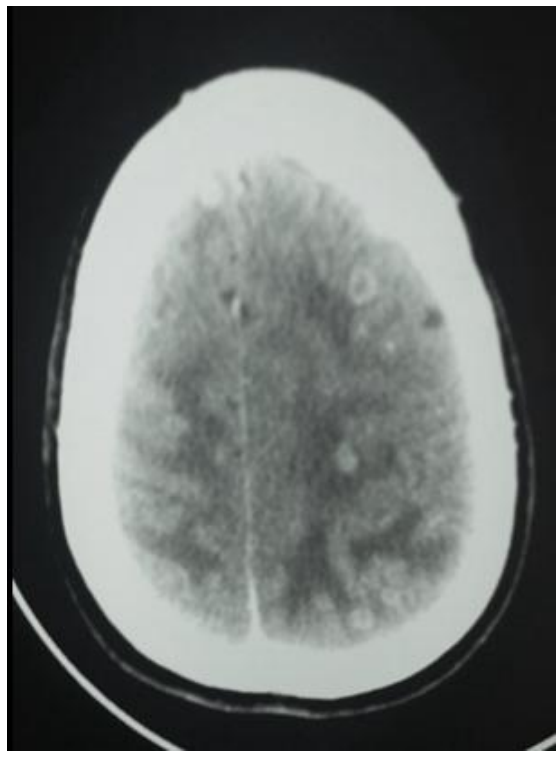

Fig-1: Cerebral CT scan showing multiple brain tuberculomas with peri-lesional edema

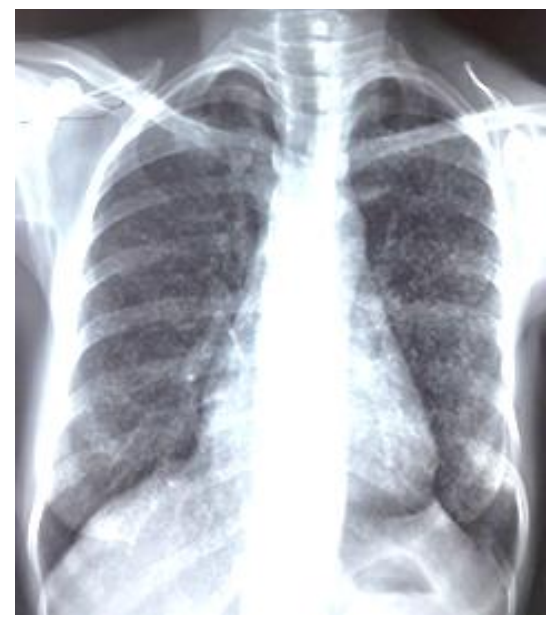

Fig-2: Chest X-ray showing a miliary appearance with rectitude of the left border of the heart related to tuberculous pericarditis

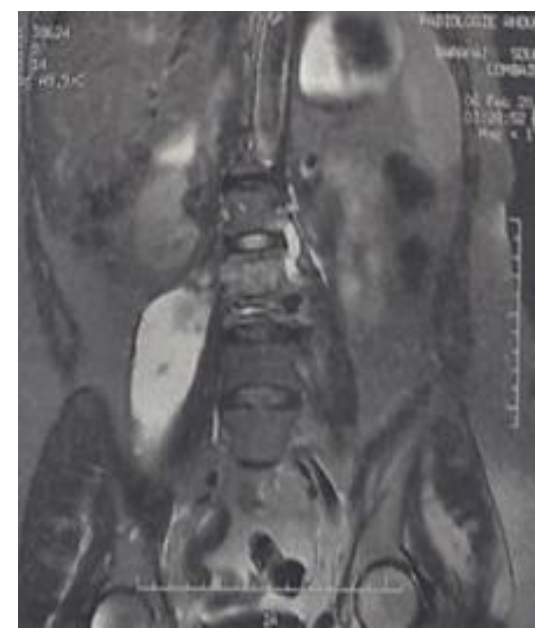

Fig-3: Lumbar MRI showing L2L3 spondylodiscitis associated with psoas abscess. 


\section{DISCUSSION}

Although the tuberculosis death rate has decreased by $37 \%$ between 2000 and 2016, it remains the ninth leading cause of death in the world and the leading cause of death due to a single infectious agent in front of HIV / AIDS [1]. Extrapulmonary forms represent 20 to $40 \%$ depending on the series. Among extrapulmonary disorders, multifocal lesions account for $10 \%$ [3-5] and occur most often in immunocompromised patients and especially those infected with HIV. Morbidity and mortality remain high. The field further aggravates the prognosis with a mortality rate of 16 to $25 \%$ [2].

In our series, patients did not present any particular terrain. They all benefited from a report in search of an underlying immunosuppression that proved to be negative. The spread of lesions can be explained by the delay in diagnosis that is often reported in African studies. In our patients the average time between the onset of symptoms and the first consultation was relatively long 72 days, probably related to the low socio-economic and cultural level of the patients. Undernutrition is also a risk factor for multifocal tuberculosis [6,7].

Thus Cathérinot described the syndrome of Mendelian susceptibility to mycobacterial infections (SSMIM) by the existence of a defect of the axis interleukin 12-interferon gamma, responsible for the occurrence of disseminated or recurrent tuberculosis despite the absence of favoring factors [8].

The concept of recent tuberculous contagion is found in $33 \%$ of our patients. In an endemic country like Morocco, a source of contagion almost always exists, but is often not reported by the patient.

After pulmonary involvement, the pleural and ganglionic localization were the most frequently observed in our series as well as in the various series of the literature $[9,10]$, easily contributing to the diagnosis by its accessibility to the anatomo-pathological examination. Peritoneal and pericardial involvement were observed respectively in $19.4 \%$ and $8.9 \%$ of cases. These figures remain lower than those observed in the other series $[11,12]$. Splenic tuberculosis involved ten patients. It remains one of the rare aspects of hematopoietic tuberculosis, even in countries with a high TB endemic such as ours $[13,14]$.

The urogenital tuberculous localization is associated with extrapulmonary disseminated disease in $18 \%$ of non-immunocompromised patients [15]. It is characterized by an often insidious but destructive evolution, resulting in irreversible sequels. Hence the need to systematically search for this location to avoid often daunting complications including kidney failure. Osteoarticular involvement was noted in $7.4 \%$ of our patients, which is consistent with figures reported in the literature [16]. It is a location often under-diagnosed especially if it is inaugural sometimes explaining the diagnostic delay. Cerebro-meningeal tuberculosis was observed in six patients, five of whom had tuberculous miliary disease. This is the most serious form. The diagnostic confirmation is based on a set of epidemiological, clinical, biological and radiological arguments given the lack of sensitivity of the diagnostic means. Direct examination and culture of cerebrospinal fluid are only positive in 10 to $30 \%$ of cases [17]. Cutaneous tuberculosis interested two cases in our series. This form represents $2.1 \%$ of the localizations in the literature and remains a form with easy diagnosis [18]. Tuberculosis of the cavum is a rare localization, described by GRAFF in 1936 [19]. It concerned two patients in our series. Psoas abscess is a rare but known localization of tuberculosis. It is most often secondary to Pott's disease, which dissects the surrounding tissues and forms an abscess by flowing along the psoas. , more rarely to other intra- or retroperitoneal disorders, particularly digestive and urogenital ones.

Multifocal tuberculosis is usually serious and may involve functional or life-threatening prognosis, requiring early and prolonged treatment in order to avoid sometimes formidable complications. Our patients were treated according to the recommendations of the national program for the fight against tuberculosis in Morocco, which is based on a poly antituberculous chemotherapy. It uses four major antibacillary drugs: isoniazid $(\mathrm{H})$, rifampicin $(\mathrm{R})$, pyrazinamide (PZA) and streptomycin (S) which has been replaced by ethambutol (E) from 2011. The evolution under treatment was favorable in the majority of our patients is $68.6 \%$ of cases. The mortality rate in our series was $3 \%$. This figure remains lower than that reported in the literature which is 16 to $25 \%$ [2]. This difference could be explained by the peculiarity of our patients not presenting immunodeficient ground and also by the introduction of the DOTS strategy. Promiscuity and the low socioeconomic level are the main risk factors favoring the occurrence of tuberculosis in our context. Improvement of the living conditions of people at risk, health education, screening of those with close contact with a contagious case, treatment of sources of contamination and BCG vaccination are the essential means of combating this serious form of tuberculosis.

\section{CONCLUSION}

Through our study, we emphasize that an immunocompetent subject without risk factors can present a severe and multifocal form of tuberculosis. Also we insist on the systematic search for certain forms such as the urogenital or pericardial attack characterized by an insidious evolution, leading to irreversible long-term sequelae. The prognosis remains relatively good subject to early and prolonged treatment. We insist on prevention based on BCG vaccination and improving living conditions. 


\section{REFERENCES}

1. WHO Report on the fight against tuberculosis in the world ; 2017.

2. Denis-Delpierre N, Merrien D, Billaud E, Besnier JM, Duhamel E, Hutin P. Multifocal tuberculosis: about 49 cases. Patho Biol. 1998; 46: 375-9.

3. Elouazzani H, Bouchentouf R, Rguibi M, Rhorfi I, Ouarssani A, Yassir Z. Multifocal tuberculosis: fatal outcome due to toxic drug reactions. Rev Pneumol Clin. 2002. Feb; 58 (1): 39-42.

4. Stelianides S, Belmatoug N, Fantin B. Manifestations and diagnosis of extrapulmonary tuberculosis Rev Mal Respir. 1997 Dec; 14 Suppl 5: S72-87

5. Mjid M, Cherif J, Ben Salah N, Toujani S, Ouahchi Y, Zakhama H. Epidemiology of tuberculosis. Rev Pneumol Clin. 2015 Apr-Jun; 71 (2-3): 67-72.

6. Ekingen BG, Guvenc H., Kahraman H. Multifocal Tuberculosis of the Chest Wall without Pulmonary Involvement. Acta Chir Belg. 2006 Jan-Feb; 106 (1): 124-6.

7. Davies PD. Risk factors for tuberculosis. Monaldi Arch Chest Dis. 2005 Mar; 63 (1): 37-46.

8. Catherinot E, Fieschi C, Feinberg J, Casanova JL, Gouderc LJ. Genetic susceptibility to mycobacterial disease: Mendelian disorders of Interleukin-12 Interferon-axis. Rev Mal Respir. Nov 2005; 22 (5 pt 1): 767-76.

9. Mazza-Stalder J, Nicoda L, Janssens JP. Extrapulmonary tuberculosis. Rev Mal Respir. 2012; 29 (4), 566-578.

10. Ghorbel H, Ben Arab N, Maaloul I. Infectious, 3029 Sfax, Multifocal tuberculosis: study of 47 cases, UHC Hedi Chaker, Tunisia Disease Service. Med Mal Infect. 2008; 38, S184-S185.

11. Hammi S, Idahmed I, Jahnawi N, Marc K, Soualhi M, Zahraoui R. Multifocal tuberculosis in 46 cases, pneumology department, Moulay-Youssef hospital, CHU, Rabat, Morocco, Rev Mal Respir. 2012; S1A129.

12. Oubulmane N, Amro L, Ait Batahar S: Multifocal tuberculosis in the subject Immunocompetent FMPM, PCIM research laboratory, Marrakech, Morocco, Rev Mal Respir. 2012; 29, S1-A209.

13. Berady S, Rabhi M, Bahrouch L. Pseudotumor isolated splenic tuberculosis: About a case. Rev Med Int. 2005; 26: 588-91.

14. Rhazal F, Lahlou MK, Benamer S, Daghri JM, Essadel E, Mohammadine E. Splenomegaly and splenic pseudotumor due to tuberculosis: six new cases. Ann Chir. 2004 Oct; 129 (8): 410-4.

15. Wise GJ. Urinary tuberculosis: modern issues. Curr Urol Rep. 2009 Jul; 10 (4): 313-8.

16. Ben Abdelghani K, Mahfoudhi M. Multifocal tuberculosis in immunocompetent subjects: two cases. Rev Rheumatism. 2006; 73 (10-11): 1122.

17. B. Kilani, L. Ammari, H. Tiouiri. Initial neuroradiological manifestations of central nervous system tuberculosis in adults: About 122 cases. Rev Med Int. 2003; 24: 86-96.

18. Fenniche S, Ben Jennet S, Marrak H, Khayat O, Zghal M, Ben Ayed M. Cutaneous tuberculosis: anatomical and clinical features (26 cases) Ann Dermatol Venereol. 2003 Nov; 130 (11): 1021-4.

19. GM Tse, Ma TK, Chan AB, FN Ho, King AD, Fung KS. Tuberculosis of the nasopharynx: a rare entity revisited. Laryngoscope. 2003 Apr; 113 (4): 737-40. 